\title{
Mixed Mode, Sequential and Relaxation Oscillations in the Belousov-Zhabotinsky System
}

\author{
Małgorzata Rachwalska \\ Faculty of Chemistry, Jagiellonian University, ul. Ingardena 3, 30-060 Kraków, Poland \\ Reprint requests to M. R.; E-mail: Rachwals@ chemia.uj.edu.pl
}

Z. Naturforsch. 62a, 41 - 55 (2007); received October 16, 2006

\begin{abstract}
The behaviour of a system composed of malonic acid (MA), $\mathrm{KBrO}_{3}, \mathrm{H}_{2} \mathrm{SO}_{4}$, and ferroin was investigated in batch experiments at various concentrations of oxygen above the chemical mixture when changing the concentration of MA. We could observe that at $10 \%$ of oxygen or more and for initial concentrations of malonic acid $[\mathrm{MA}]_{0}$ between $0.15 \mathrm{M}$ and $0.6 \mathrm{M}$ the system attains an equilibrium by some of mixed mode oscillations. Such a behaviour of the system could be described by a model used lately. Additionally for $[\mathrm{MA}]_{0}=0.1 \mathrm{M}$ or less at $0-20 \%$ of oxygen a region of sequential oscillations was discovered (observed for the first time in the system with ferroin) and a trial of understanding of the event is suggested. For rather small $[\mathrm{MA}]_{0} \mathrm{ca}$. $0.025 \mathrm{M}$ no mixed mode oscillations, no sequential ones but the so-called relaxation oscillations were observed.
\end{abstract}

Key words: Belousov-Zhabotinsky System; Mixed Mode; Sequential and Relaxation Oscillations.

\section{Introduction}

Recently [1] we have studied the oxygen effect on the time-dependent bifurcations of transient oscillations in the Belousov-Zhabotinsky (BZ) oscillating chemical reaction in a closed system. We showed there only a small part of a phase diagram (concentration of oxygen in its mixture with nitrogen above the surface of the chemical mixture against initial concentration of malonic acid $[\mathrm{MA}]_{0}$ ). Namely, some results for $[\mathrm{MA}]_{0}=0.68 \mathrm{M}$ were presented for various portions of oxygen. Experiments discussed [1] showed that oscillations disappeared through different bifurcations depending on the oxygen concentration in the gas phase above the reaction solution. Oscillations disappeared through the time-delayed Hopf bifurcation at low oxygen contents (till ca. 28\%), whereas at high oxygen contents (greater or equal to $30 \%$ ) they disappeared through the time-dependent Sniper (saddle-node infinitive period) bifurcation.

The aim of the work here is to discuss some results of that phase diagram for $[\mathrm{MA}]_{0}$ less than $0.68 \mathrm{M}$ in order to compare a process of oscillations disappearing with those presented earlier [1].

In the region of $[\mathrm{MA}]_{0}=0.15-0.6 \mathrm{M}$, some mixed mode oscillations (MMOs) were obtained, for a portion of the oxygen-nitrogen mix above the surface of the reaction mixture (ca. 0.05-0.3, depending on
$[\mathrm{MA}]_{0}$, to 1.0). In such conditions some MMOs were obtained. The system attained an equilibrium in the manner of Sniper bifurcation, which was carefully discussed [1]. The chemical reactions, used by us in our previous article [1] to describe Hopf and Sniper bifurcations, were not able to reproduce the mixed mode region, but the simple theoretical model $[2,3]$ used previously [4] has done it quite well and it is shown in the work.

The second problem discussed in the work is connected with the so-called sequential oscillations. The problem of some sequential oscillations was discussed in many articles [5-9] dealing with BZ systems having only one or many organic substances in batch conditions.

The authors of [5] for example considered how the $\mathrm{BZ}$ reaction might behave if more than one organic substrate is present. They state that possible modes of behavior include: A) inhibition of oscillation; B) independent concurrent oscillations perceived for example as beats, modified wave forms or chaotic fluctuations; C) entrainment, i. e. oscillations generated by a single substrate which "drives" the other substrate reactions to follow the dominant rhythm, resulting possibly in enhanced oscillation amplitudes; D) sequential oscillations, in which the system first uses one substrate almost completely and then, perhaps after a transition period, consumes the next substrate. 
Such a system, i. e. with two organic substrates, was considered for example in [6]. In the course of their investigations of the oxalic acid/acetone mixed substrate BZ system, Wittmann et al. observed a pause of oscillations and an induction period depending on the initial composition. The length of the first oscillating period decreased with an increasing initial acetone concentration until the pause turned into an induction period. Both kinds of quiescent periods were excitable by bromide ions. The effect of $\left[\mathrm{Br}^{-}\right]$on the behaviour of a system showing some sequential oscillations was also shown [8]. The ferroin-catalyzed BZ-type reaction with 3,4-dihydroxybenzoic acid as organic substrate showed two types of temporal oscillations depending on the initial concentration of bromide. When $\left[\mathrm{Br}^{-}\right]$was very high, damped highfrequency oscillations appeared. When $\left[\mathrm{Br}^{-}\right]$was very low, low-frequency oscillations of the normal type were obtained. At moderate concentrations of bromide both high-frequency and low-frequency oscillations could be monitored. Effects of temperature on high-frequency and low-frequency oscillations were similar. The oscillation frequencies increased with increasing temperature. More discussion regarding the chemistry of some sequential oscillations in the system $\mathrm{MA}, \mathrm{NaBrO}_{3}, \mathrm{H}_{2} \mathrm{SO}_{4}, \mathrm{CeNH}_{4}\left(\mathrm{NO}_{3}\right)_{4}$ is given in [7]. At several initial concentrations of the substances [7], some oscillations were observed at the very beginning of the reaction followed by no oscillations and again some oscillations. The first group of oscillations was radical-controlled and the second group was bromide-controlled. Radical-controlled oscillations were seen with or without an induction period. In the first case higher $\left[\mathrm{H}_{2} \mathrm{SO}_{4}\right]$ was used and also acetylacetone. Bromide-controlled, radicalcontrolled or a combination of both bromide- and radical-controlled oscillations could be obtained by varying the MA or the sulfuric acid concentration. Those sequential oscillations are also easily obtained by use of a chemical reaction simulation model where some of the reactions include radicals such as MA. and $\mathrm{BrO}_{2}$.

Sequential oscillations can be associated with the switching on of a second oscillator after preparation of a substrate (used later by the second oscillator) in the first part of oscillatory behaviour of a system [9]. In [10] only one organic substrate, namely for the uncatalyzed system: $\mathrm{BrO}_{3}{ }^{-}$, phenol, $\mathrm{H}_{2} \mathrm{SO}_{4}$ was used. Such uncatalyzed systems have also been investigated in [11].
Some sequential oscillations presented in this article were discovered accidentally when preparing the phase diagram mentioned above (portion of oxygen in its mixture with nitrogen over a chemical mixture composed of $\mathrm{MA}, \mathrm{KBrO}_{3}$, ferroin, $\mathrm{H}_{2} \mathrm{SO}_{4}$ against initial concentration of $\left.[\mathrm{MA}]_{0}\right)$. So far as we know it was the first time that some sequential oscillations could be observed in the chemical system with ferroin. When studying the phase diagram one could see that for small initial concentrations of malonic acid, less or equal to ca. $0.1 \mathrm{M}$, there appeared some sequential oscillations in the system with nitrogen flowing over the mixture. The number of those oscillations decreased when a flowing agent composed of a mixture of nitrogen and oxygen was used. The last effect (called "decreasing") is explained by many articles $[1,11]$ which describe the loss of oscillations in the presence of oxygen.

Independently, one can ask the question: what is the reason for the appearance of sequential oscillations in the investigated system? Some investigations of the phase diagram discussed above showed a region of initial concentrations of malonic acid which is useful for finding some sequential oscillations, namely ca. $0.1 \mathrm{M}$, or less $[\mathrm{MA}]_{0}$, as mentioned above. Some questions are existing at the very beginning of the experiments related to the dependency of the form of sequential oscillations on initial concentrations of malonic acid, on the concentration of ferroin, on the temperature, on the atmosphere over a mixture, on the flow or non-flow of a gas over the mixture (static or dynamic conditions) and on the composition of the gas. One could expect that an experimental answer to these questions would give some estimations of the nature of some of the sequential oscillations, particularly those appearing in the system as well as the nature of some relaxation oscillations which were observed for very small $[\mathrm{MA}]_{0}$.

\section{Experimental}

\subsection{Reagents}

Analytical grade chemicals, $\mathrm{KBrO}_{3}, \mathrm{H}_{2} \mathrm{SO}_{4}, 1,10$ phenanthroline ("POCH" S. A. Gliwice) and malonic acid (Aldrich), were used without further purification. Stock solutions of $\mathrm{KBrO}_{3}, \mathrm{H}_{2} \mathrm{SO}_{4}$, and MA were prepared using doubly distilled water. Aqueous ferroin solution was prepared using the standard method [12].

\subsection{Apparatus}

A thermally insulated cylindrical glass vessel was used in all experiments (internal dimensions: diame- 


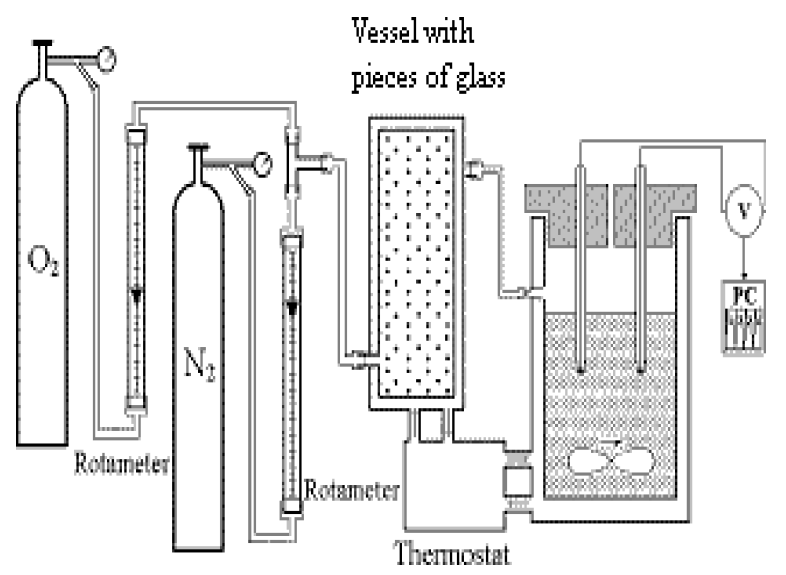

Fig. 1. Experimental set up.

ter $37 \mathrm{~mm}$, height $110 \mathrm{~mm}$ ), and a rod-shaped tefloncoated magnetic stirrer (length $21 \mathrm{~mm}$, diameter $5 \mathrm{~mm}$ ) was applied. Some details of the experimental set up are shown in Figure 1. The stirring rate was $740 \mathrm{rpm}$. In only one case the stirring rate was not equal to $740 \mathrm{rpm}$, but it was equal to $900 \mathrm{rpm}$. Experiments were generally performed at a constant temperature of $24.5^{\circ} \mathrm{C}$, although, some experiments were carried out at other temperatures. The volume of the entire reaction mixture was $40 \mathrm{ml}$. The reaction was followed by measuring and recording the potential of a platinum and bromide electrode, using a saturated calomel reference electrode connected with the reaction mixture by a salt bridge with $1 \mathrm{M} \mathrm{KNO}_{3}$. Changing potentials vs. time were recorded by an MTA (Kutesz, type 1040/4) recorder and a PC. Above the surface of the reaction mixture there was a gaseous mixture containing oxygen and nitrogen in a specific proportion at a pressure of $1 \mathrm{~atm}$. Some experiments were carried out in air (no flowing nitrogen), in flowing air and in flowing oxygen.

\subsection{Procedures}

In our batch experiments the reaction was initiated by adding the ferroin solution into the mixture of $\mathrm{KBrO}_{3}, \mathrm{MA}, \mathrm{H}_{2} \mathrm{SO}_{4}$. In all experiments the initial concentrations of the reagents were identical: $\left[\mathrm{KBrO}_{3}\right]_{0}=0.19 \mathrm{M}$, $\left[\mathrm{H}_{2} \mathrm{SO}_{4}\right]_{0}=0.32 \mathrm{M}, \quad[\text { ferroin }]_{0}=0.00338 \mathrm{M}$ (or $0.00188 \mathrm{M}$ in some cases) and $[\mathrm{MA}]_{0}$ varied from $0.6 \mathrm{M}$ to $0.025 \mathrm{M}$. Before adding the ferroin

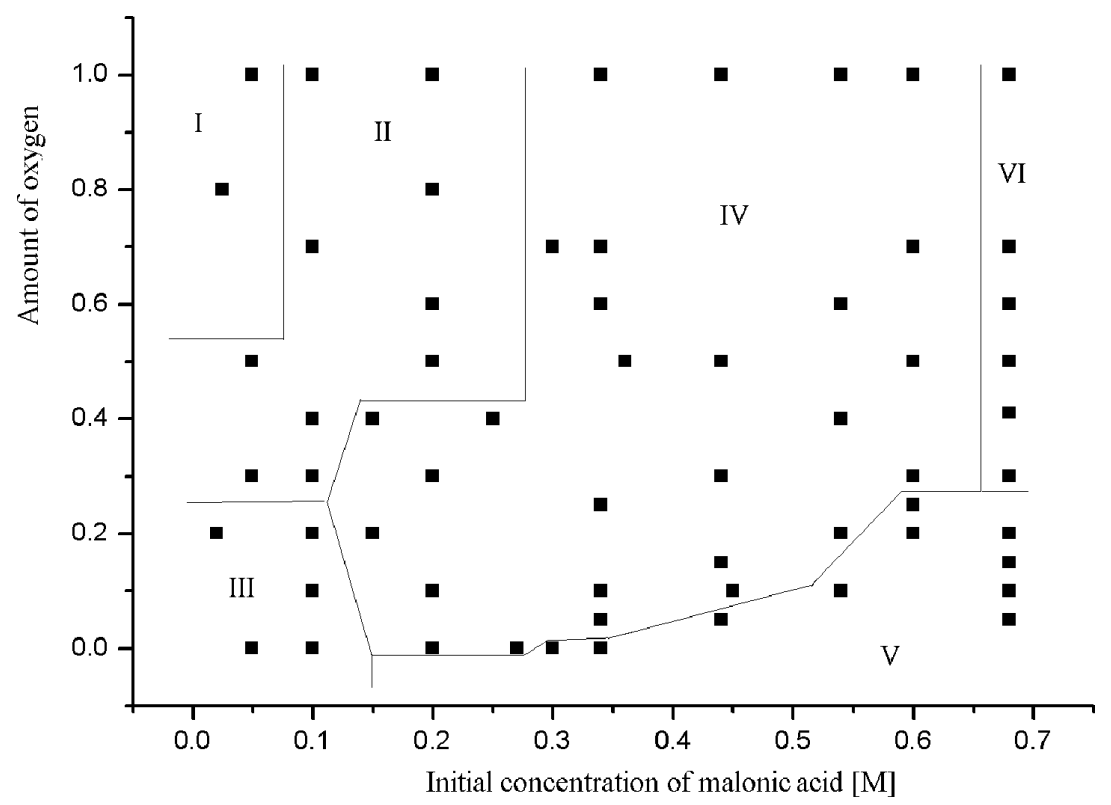

Fig. 2. Phase diagram: portion of oxygen in its mixture with nitrogen against initial concentration of malonic acid. I - Region of stable state (no oscillations in $30 \mathrm{~min}$ ). II - Oscillations disappear by Sniper bifurcations. III - Region of sequential oscillations (and the region of relaxation oscillations for very small $[\mathrm{MA}]_{0}$ as for example $0.025 \mathrm{M}$ ). IV - Oscillations disappear by Sniper bifurcations but in contrast to the regions I and II, III, V, VI in oscillations' region there appear no pure L-type oscillations, but Ls1-type. Therefore we call the region IV region of mixed mode oscillations. V - Oscillations disappear by Hopf bifurcations. VI - Oscillations disappear by Sniper bifurcations as in region II, but the time of reaching equilibrium (stable state in some experimental conditions) is in the case of region VI much longer than in the case of region II. 


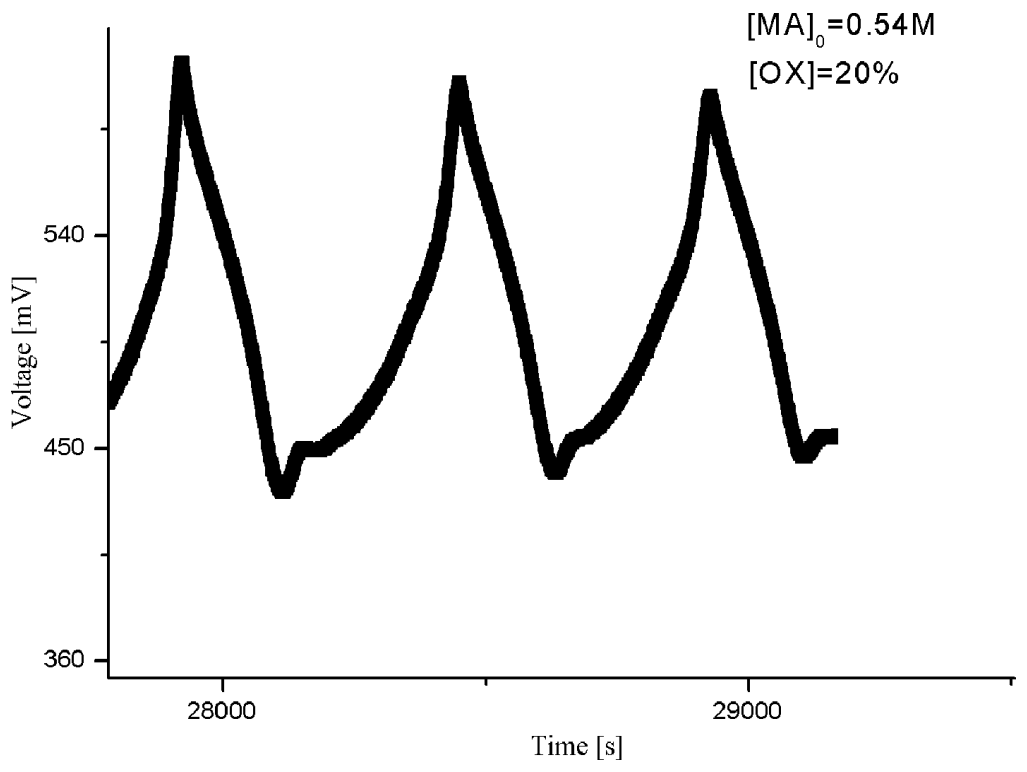

Fig. 3. An experimental example of mixed mode oscillations. solution into the reaction mixture, during ca. $30 \mathrm{~min}$, the oxygen-nitrogen mixture (or only nitrogen or only oxygen or air) was allowed to flow over the reaction mixture at a velocity of $100 \mathrm{l} / \mathrm{h}$ to stabilize the gas phase in the reactor. The gas was first passed through a glass vessel filled by small pieces of glass to provide sufficient mixing of the gases. Pressure of the gasses was controlled by rotameters.

\section{Results}

\subsection{Mixed Mode Oscillations}

Figure 2 shows the phase diagram and an example of some mixed mode oscillations is shown on Figure 3. At the end of some experimental runs a deformation of the oscillation peaks appeared as can easily be seen in Figure 4. The resulting formation is a shoulder. In many other cases a distinct Ls1 pattern was obtained.

The simple three-variable model [2,3] which qualitatively describes various asymptotic MMO observed in the BZ reaction, has been studied recently. This model was also helpful in the successful searches of new types of MMO with $\mathrm{LS}_{\mathrm{n}} \mathrm{s}_{\mathrm{m}}$ [4] patterns:

$$
\begin{gathered}
\frac{\mathrm{d} v}{\mathrm{~d} t}=r\left[u-\left(v-v_{1}\right)\left(v-v_{2}\right)\left(v-v_{3}\right)-a\right]=r f(u, v), \\
\frac{\mathrm{d} u}{\mathrm{~d} t}=b-b_{1}-b_{2} v-u=g(u, v)
\end{gathered}
$$

$$
\frac{\mathrm{d} p}{\mathrm{~d} t}=q(v-p) .
$$

Assume that the parameters in (1) - (3) are kept constant and have the values $v_{1}=10, v_{2}=11, v_{3}=20$, $a=150, b=436.6, b_{1}=3.714, b_{2}=21.7$ and $q=$ 0.215 . For $r$ (bifurcation parameter) equal to 0.081 we get the pattern Ls1 (Fig. 5) which can roughly describe some experimental results (Fig. 3).

\subsection{Experimental Runs}

Let us recall some experiments described briefly above, not published yet. They were performed at a stirring rate equal to $740 \mathrm{rpm}$ and are gathered in items 1) and 2) below. Some experimental runs were performed at $24.5{ }^{\circ} \mathrm{C}$ (maintained by circulating water) in a batch system for the following initial concentrations: $[\text { ferroin }]_{0}=0.00338 \mathrm{M},\left[\mathrm{KBrO}_{3}\right]_{0}=0.19 \mathrm{M}$, $\left[\mathrm{H}_{2} \mathrm{SO}_{4}\right]_{0}=0.32 \mathrm{M}$ :

1) Nitrogen flowing over the surface of the chemical mixture (designated in some figure captions) a) for various initial concentrations of malonic acid $(0.025 \mathrm{M}$, $0.04 \mathrm{M}, 0.05 \mathrm{M}, 0.06 \mathrm{M}, 0.1 \mathrm{M})$ and other initial concentrations as above; b) for various initial concentrations of malonic acid $(0.025 \mathrm{M}, 0.05 \mathrm{M}, 0.07 \mathrm{M}, 0.08 \mathrm{M}$, $0.09 \mathrm{M}$ ) and [feroin $]_{0}$ equal to $0.00188 \mathrm{M}$ different than in previous experiments, $\left[\mathrm{KBrO}_{3}\right]_{0}$ and $\left[\mathrm{H}_{2} \mathrm{SO}_{4}\right]_{0}$ as above. Some measurements for $[\mathrm{MA}]_{0}=0.08 \mathrm{M}$ were performed at various temperatures with flowing nitrogen and additionally in air. 


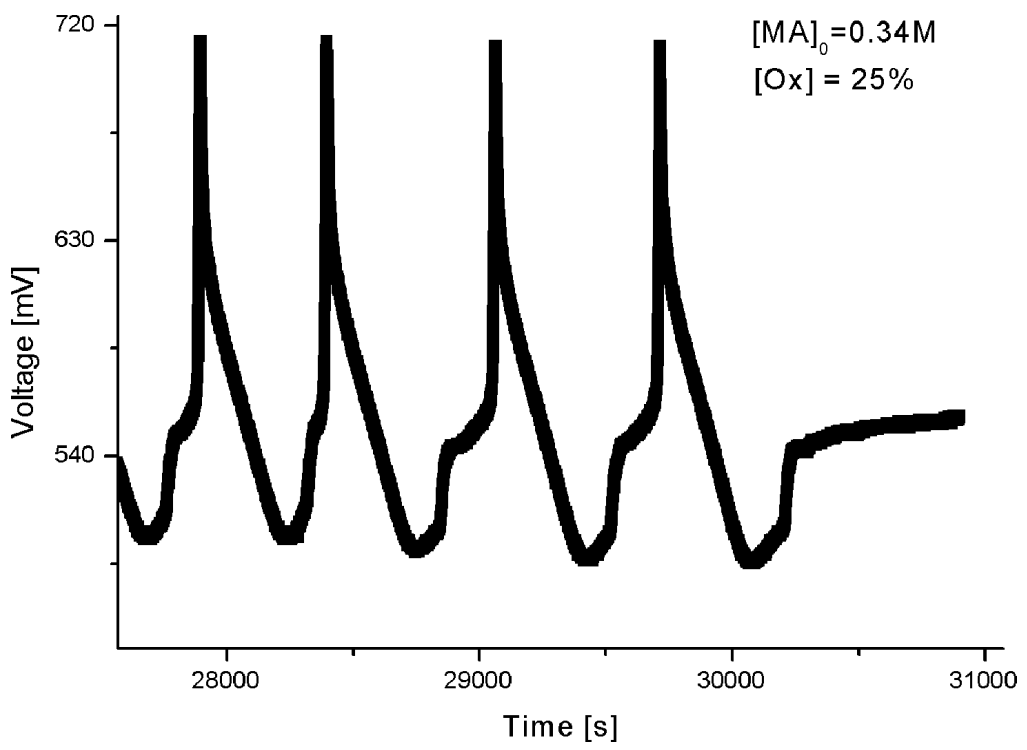

Fig. 4. Mixed mode oscillations shown as shoulders.

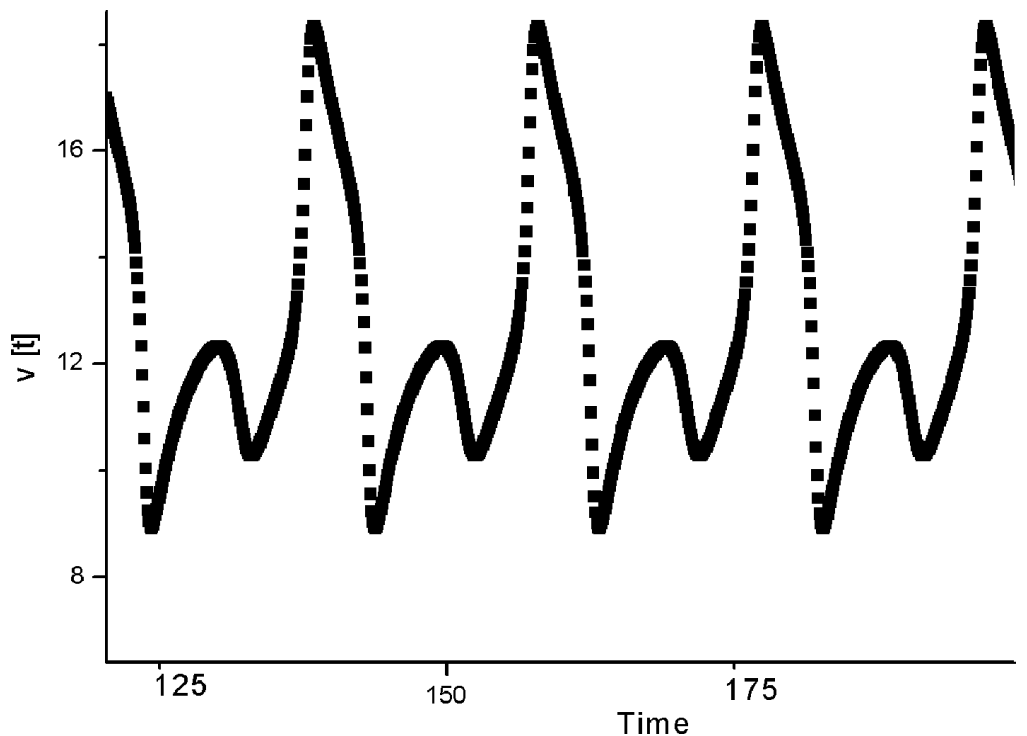

Fig. 5. A theoretical pattern imitating an experimental one (from Fig. 3).
2) For $[\mathrm{MA}]_{0}$ equal to $0.025 \mathrm{M}$ and $0.03 \mathrm{M}$ and [ferroin $]_{0}$ equal to $0.00388 \mathrm{M}$ with electrodes inserted into the reactor in a way different than in all other experiments. Namely in the experiments electrodes were placed in a cover, usually used in CSTR measurements. During measurements the cover was plunged into the investigated chemical mixture.

3) Some experiments were performed in air, i.e. in a batch reactor which was not isolated from the atmosphere. The batch reactor was open to the atmosphere through holes in the electrodes' holder, for $[\text { ferroin }]_{0}$ equal to $0.00338 \mathrm{M}$ and $[\mathrm{MA}]_{0}=$
$0.025 \mathrm{M}$ at various temperatures in air and with flowing nitrogen.

4) Some experiments were performed for $[\mathrm{MA}]_{0}$ equal to $0.05 \mathrm{M}$, [ferroin $]_{0}=0.00338 \mathrm{M},\left[\mathrm{KBrO}_{3}\right]_{0}=$ $0.19 \mathrm{M},\left[\mathrm{H}_{2} \mathrm{SO}_{4}\right]_{0}=0.32 \mathrm{M}$ in air, with flowing nitrogen (at various temperatures) and additionally at 24.5 ${ }^{\circ} \mathrm{C}$ with flowing nitrogen at $900 \mathrm{rpm}$. The same concentrations were used for an experiment with flowing air, oxygen over the surface of the solution.

5) Some experiments were performed for the conditions shown in the first line of Table 1 while moving an electrode during the experimental run. 

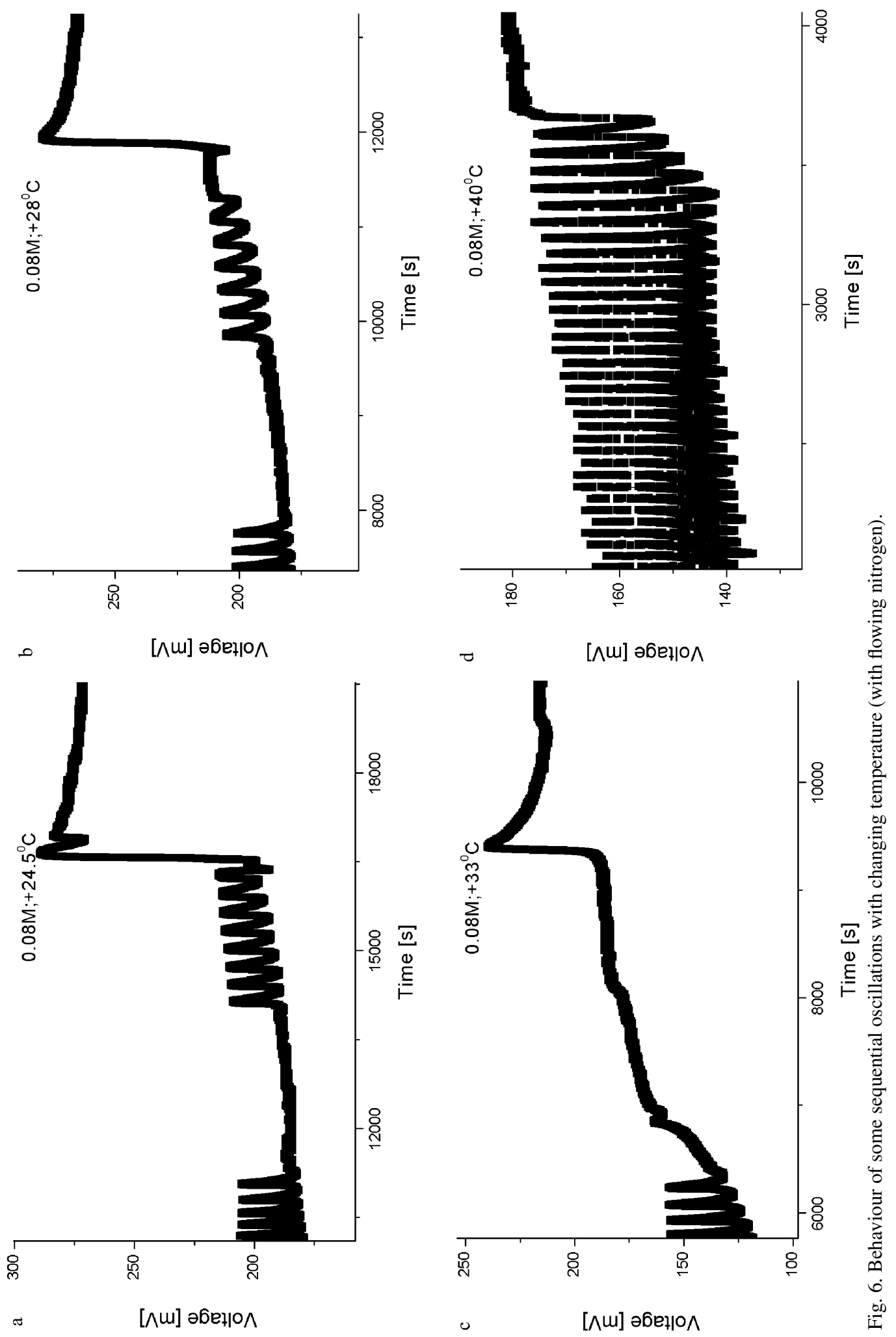
Table 1. Some important parameters for two groups of experiments giving an evidence of some sequentia.

\begin{tabular}{cccc}
\hline $\begin{array}{c}\text { ferroin }]_{0} \\
{[\mathrm{M}]}\end{array}$ & $\begin{array}{c}{[\mathrm{MA}]_{0}} \\
{[\mathrm{M}]}\end{array}$ & $\begin{array}{c}\text { Temperature } \\
{\left[{ }^{\circ} \mathrm{C}\right]}\end{array}$ & $\begin{array}{c}\text { Number of sequential } \\
\text { oscillations }\end{array}$ \\
\hline 0.00338 & 0.05 & 24.5 & 11 \\
& & 26.5 & 5 \\
& & 30.5 & 4 \\
& & 34.5 & 1 \\
- & & 40.2 & no \\
0.00188 & 0.08 & - & - \\
& & 24.5 & 10 \\
& & 33 & 8 \\
& & 40 & 2 \\
\hline
\end{tabular}

6) When changing the temperature in a small region in an experimental run for the same conditions as in 5).

\subsection{Behaviour of Some Sequential Oscillations in Various Experimental Conditions}

For $[\mathrm{MA}]_{0}=0.08 \mathrm{M}$ and $[\text { ferroin }]_{0}=0.00188 \mathrm{M}$ (the other concentrations as mentioned above) we could see that the system attains an equilibrium by the so-called sequential oscillations. After mixing the reagents at $24.5^{\circ} \mathrm{C}$, oscillations lasted till ca. $11000 \mathrm{~s}$, then through $3000 \mathrm{~s}$ they were not observed (cessation). After $14000 \mathrm{~s}, 10$ oscillations appeared again (resurrection) before equilibrium was achieved. In all experiments the conditions were kept constant within the limits of experimental error. In such a way we have described carefully one experimental run with some sequential oscillations.

In the next experimental runs, showing evidence of sequential oscillations, the situation was roughly similar. Only the time period without oscillations (cessation) was different from that mentioned above as well as the number of oscillations which appeared after the cessation. This behaviour is clearly shown in Figure 6 . At $[\mathrm{MA}]_{0}=0.1 \mathrm{M}$ some sequential oscillations were observed for oxygen levels between 0 and ca. $20 \%$. The number of sequential oscillations decreased with increasing levels of oxygen.

Some experimental runs from 1a) showed that for [ferroin $]_{0}$ equal to $0.00338 \mathrm{M},\left[\mathrm{KBrO}_{3}\right]_{0}=0.19 \mathrm{M}$, $\left[\mathrm{H}_{2} \mathrm{SO}_{4}\right]_{0}=0.32 \mathrm{M}$, it is necessary to use $[\mathrm{MA}]_{0}=$ $0.05 \mathrm{M}$ in order to get some sequential oscillations at $24.5{ }^{\circ} \mathrm{C}$ with flowing nitrogen. For [MA] $]_{0}$ smaller than $0.05 \mathrm{M}$ we did not see any sequential oscillations under the same conditions. We can not exactly say how the number of sequential oscillations (in the 'second part' of an experimental run) depends on [MA $]_{0}$ since reproducibility (in the limit of $30 \%$ ) of the number of sequential oscillations is not very good. ['Second part' refers to a group of oscillations appearing after a distinct period of an experimental run having no oscillations (a cessation). One can see that the name sequential oscillations is used for the event or only to call the second part of an experimental run.]

Results from item 1b) show that decrease in [ferroin $]_{0}$ concentration moves the region of sequential oscillations to a region greater than $[\mathrm{MA}]_{0}$. In the second part of Table 1 one can see that for [ferroin $]_{0}=$ $0.00188 \mathrm{M}$, it was necessary to have $[\mathrm{MA}]_{0}=0.08 \mathrm{M}$ to achieve some sequential oscillations. Figure 6 shows the behaviour of our system (one set of initial concentrations) with changing temperature. The number of sequential oscillations is seen to decrease with increasing temperature.

When an equilibrium is reached the electrode potentials are at high values. Such system behaviour was observed in all experimental cases. Similar behaviour was observed on both electrodes and in many cases oscillations seen on the Pt electrode ceased earlier than on the Br electrode. This behaviour will not be considered here.

Results from 1b), 2) and 4), 5), 6) show:

a) For the same initial conditions as in Table 1 (experiments performed with flowing nitrogen), but for experimental runs performed in air, increasing temperature affects the stepwise disappearance of sequential oscillations. Some experiments at $24.5^{\circ} \mathrm{C}$, showed that sequential oscillations are most distinctly shown in experiments with flowing nitrogen than in experiments performed in air. For experimental conditions from the second part of Table 110 oscillations were observed, whereas for the same initial concentrations, but for experiments performed in air, evidence of 6 sequential oscillations was observed. At $22{ }^{\circ} \mathrm{C}$ in air, only one sequential oscillation was observed. Hence we can see that decreasing temperature is connected with a decreasing number of sequential oscillations. The effect is opposite to that observed in experiments performed in flowing nitrogen (as shown in Table 1, Fig. 6), but only at a certain temperature. Finally the behaviour mentioned above is the same at higher temperatures for experiments both in nitrogen and in air.

b) The result shown in Fig. 7 was obtained [item 2)] after inserting some electrodes in a cover, whereas in all other cases the electrodes were placed in the manner normal for batch experiments. The cover inhibits escape of some gaseous products of the reaction. It was observed that between ca. 2000 and 3000 s the poten- 

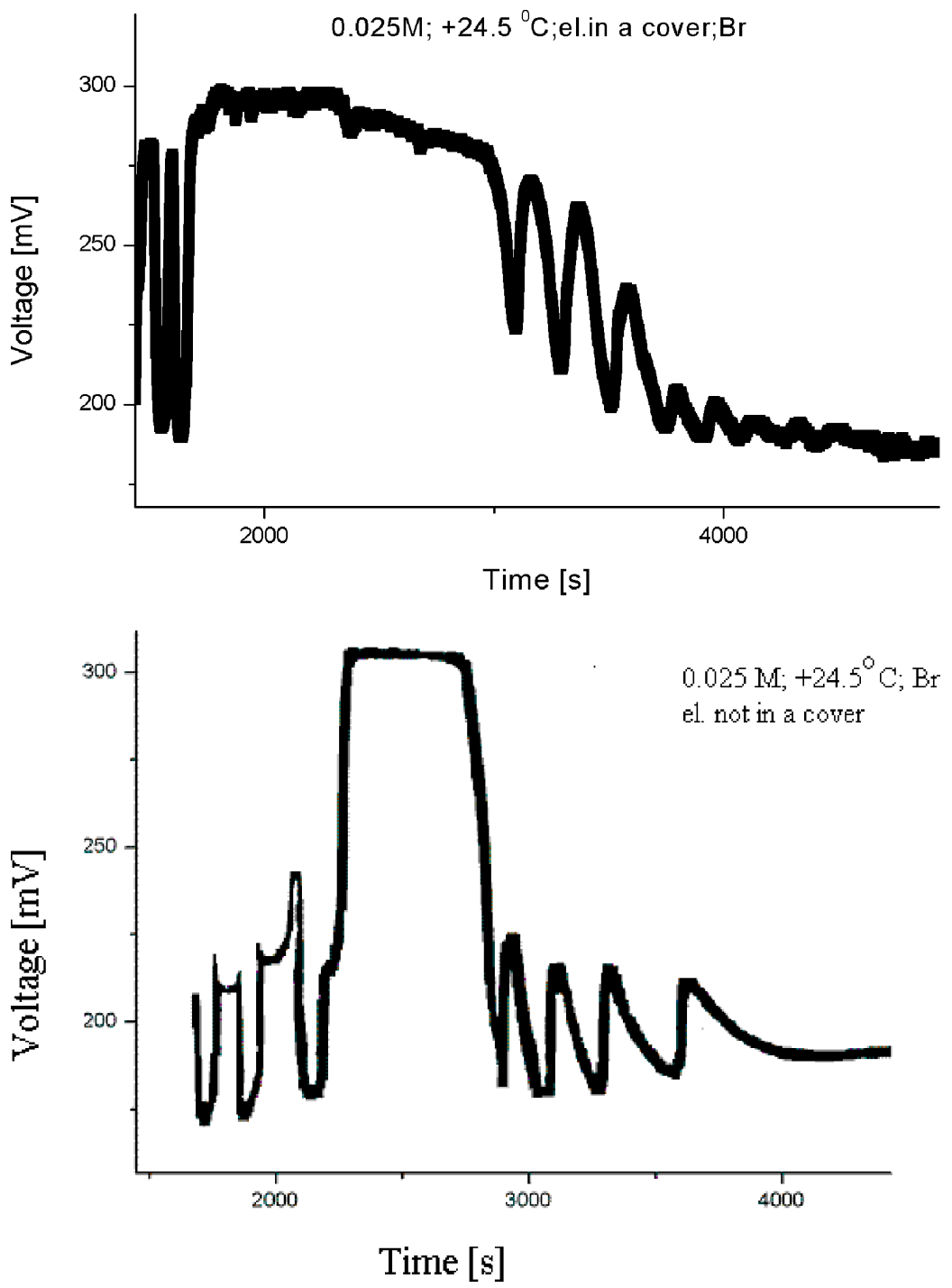

Fig. 7. The last part of oscillations in an experiment with electrodes inserted in a cover.

Fig. 8. The same experiment as in Fig. 7 but with electrodes used the "normal way" (without flowing nitrogen).

tial of the bromide electrode was high (without any oscillations) and the system continued to oscillate again till reaching an equilibrium. As the potential begun to lower, escape of some gaseous products of the reaction was easily observed in association with a portion of the chemical mixture being ejected onto the surface of the cover. Obviously, this was the reason for observation of some oscillations arising from the cessation condition. Some sequential oscillations also followed the highest value of the electrode's potential. In our previous results showing evidence of some sequential oscillations (for example see Fig. 6), the oscillations followed a lower value of the electrode's potential (where the sequential oscillations were distinctly blocked).
An experiment with the same initial concentrations, temperature, stirring rate as in Fig. 7 was performed with electrodes inserted not in a cover but the time in a normal manner (Fig. 8). We can see that Fig. 8 is similar to Fig. 7, but the time without any oscillations is ca. 2 times shorter in Figure 8. Comparing those 2 results one can easy state that really the cover with electrodes was responsible for a greater amount of gaseous products in the solution disturbing normal oscillations on the longer distance than in the experiment with electrodes inserted on a normal way.

c) Moving the Pt electrode [mentioned above in 5)] several times during an experimental run affected the results as is easily seen by comparing the experimental 

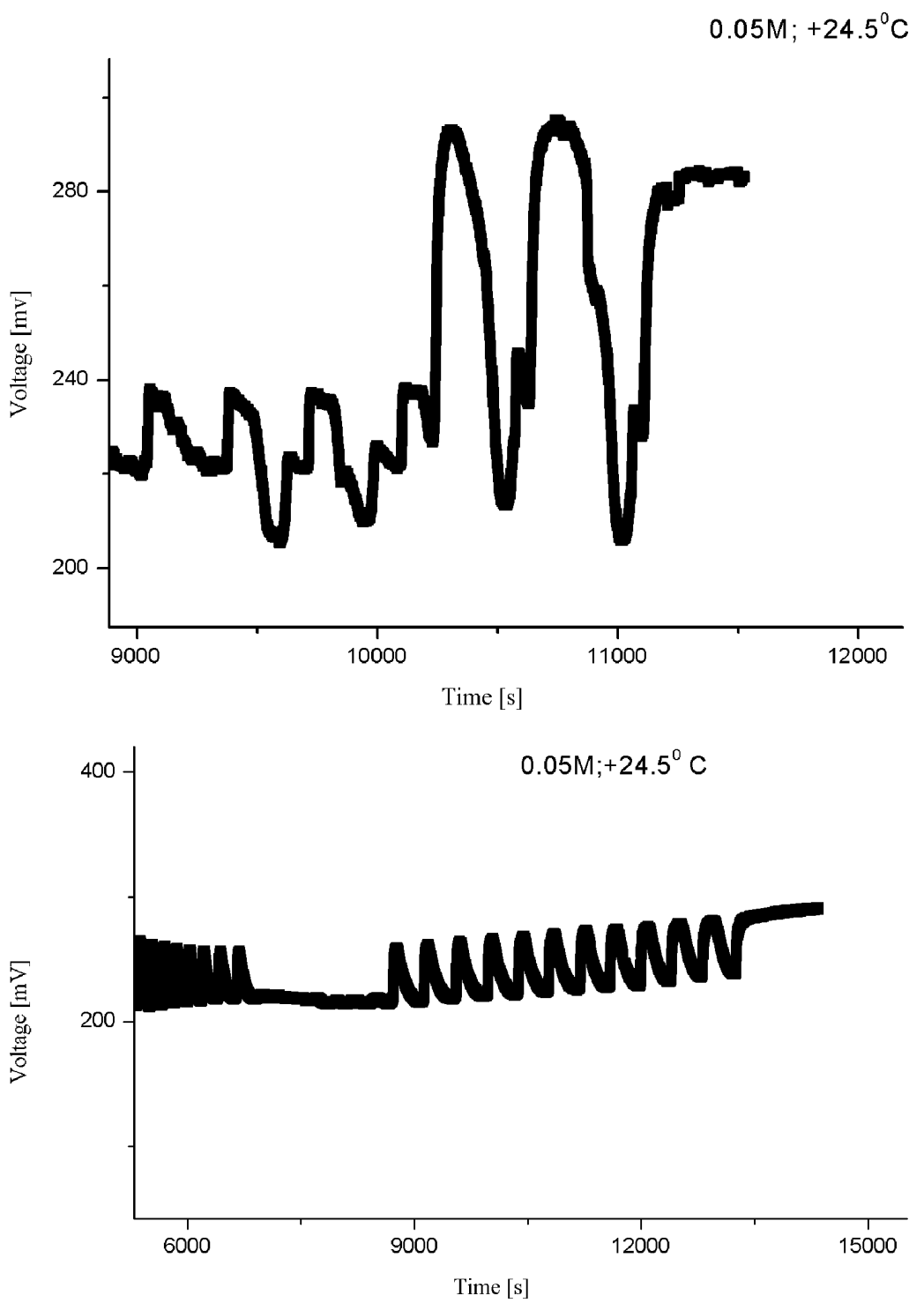

Fig. 9. Influence of electrode's moving on results (with flowing nitrogen).

Fig. 10. Results for the same experiment as in Fig. 9, but without electrode's moving. run of Fig. 9 with the next result of Fig. 10, where the Pt electrode was not moved. Such great system sensitivity was observed for the first time among many experimental runs showing oscillatory reactions. Even such complex patterns as $\mathrm{LS}_{\mathrm{n}} \mathrm{s}_{\mathrm{m}}$-type [4] were not sensitive to varying positions of electrodes during an experimental run.

d) Changing temperature [mentioned above in point 6)] over a small temperature range (designated in Fig. 11 in parenthesis) during the measurement influenced the results. Namely, no sequential oscillations were obtained in this case. In another experimental run carried out while keeping the temperature con- stant in the designated region, some sequential oscillations were obtained (of course, with other experimental conditions remaining the same, e.g. initial concentrations, stirring rate). It seems to be obvious that changing temperature, in the discussed experiment, worked as a driving force to remove gaseous products thereby influencing the shape of some peaks (increasing temperature creates narrower peaks and decreasing temperature creates broader peaks).

e) Increasing the stirring rate [to $900 \mathrm{rpm}$, point 4)] practically removed sequential oscillations. Figure 12 and 13 show a comparison of records from both electrodes at such conditions. 

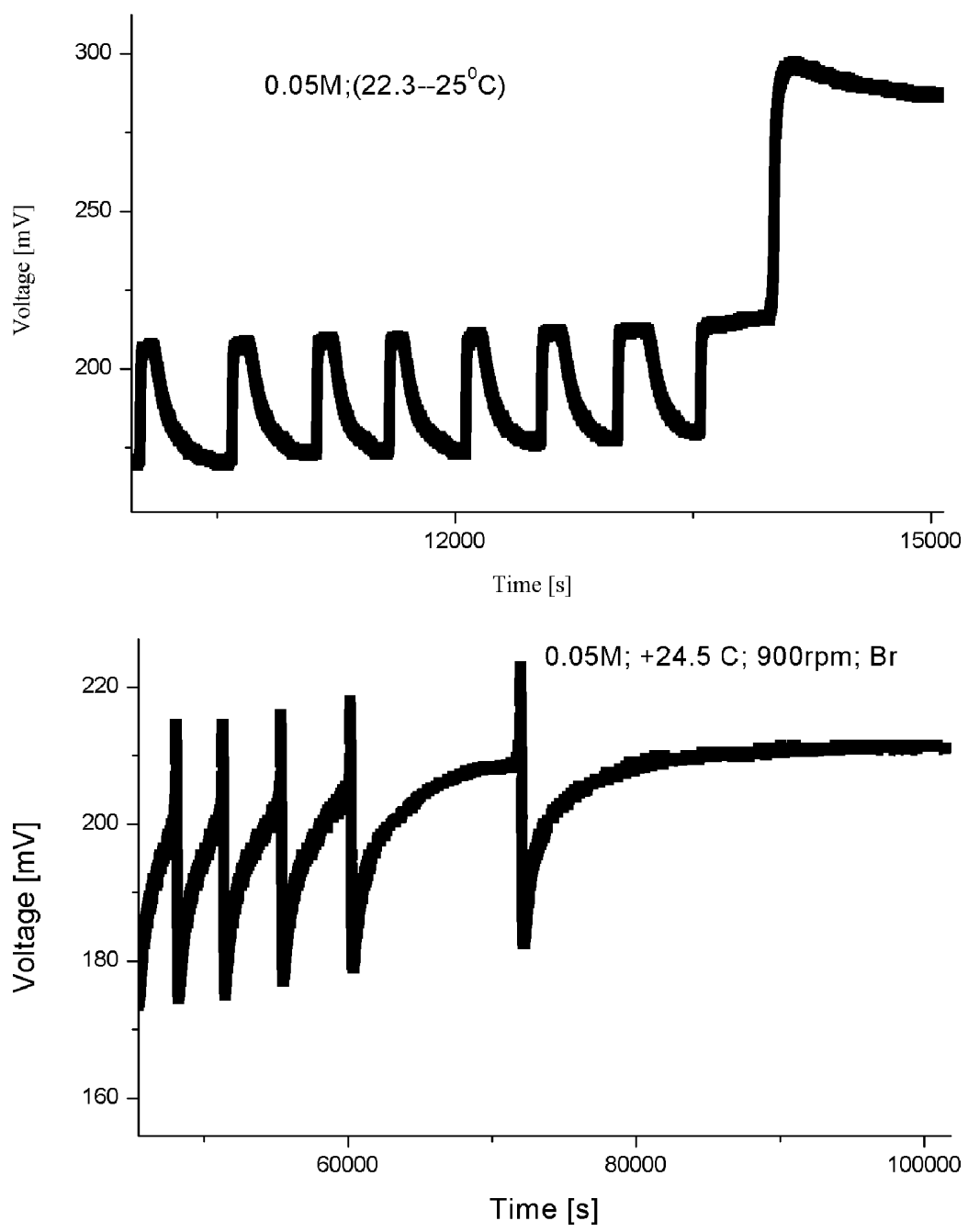

Fig. 11. Influence of small temperature change during a measurement (with flowing nitrogen).

Fig. 12. The same experiment as in Fig. 10 but for a greater stirring rate recorded using a $\mathrm{Br}$ electrode (with flowing nitrogen).

\subsection{Relaxation Oscillations}

For $[\mathrm{MA}]_{0}=0.025 \mathrm{M}[$ item 3) above $]$ there was no evidence of sequential oscillations, but an interesting way of reaching an equilibrium was observed (which seems to us to have something to do with gaseous products of the reaction dissolved in the mixture). The shape of some peaks changed in an unusual way (i. e., without any sequential oscillations), namely they were rather broad at $24.5{ }^{\circ} \mathrm{C}$ and they became slimmer (narrower) at higher temperatures (Figs. 14, 15). We believe that some gaseous products dissolved in the solution can be responsible for this behaviour, because the amount of gaseous products in the solution decreased with increasing temperature and disturbed the chemical reaction less. The total time of the reac- tion was equal to ca. $3200 \mathrm{~s}$ (with flowing nitrogen), whereas it was more than two times longer in experiments without flowing nitrogen (Figs. 16 and 17). In Fig. 18 the reproduction of the experimental run from Fig. 17 is shown. The reproduction seen when comparing Figs. 17 and 18 is rather a bad one, although in investigations of some even complex BZ systems [4] it was quite well. As we can see, although the experiments from Figs. 17 and 18 were carried out under the same conditions, their results differ one from the other. We can associate this difference with an instability of the system due to some gaseous products of the reaction being dissolved in the solution. The smallest change of the experimental conditions (even within the limits of experimental error) causes escape of such products which affects the results. In the experimental 

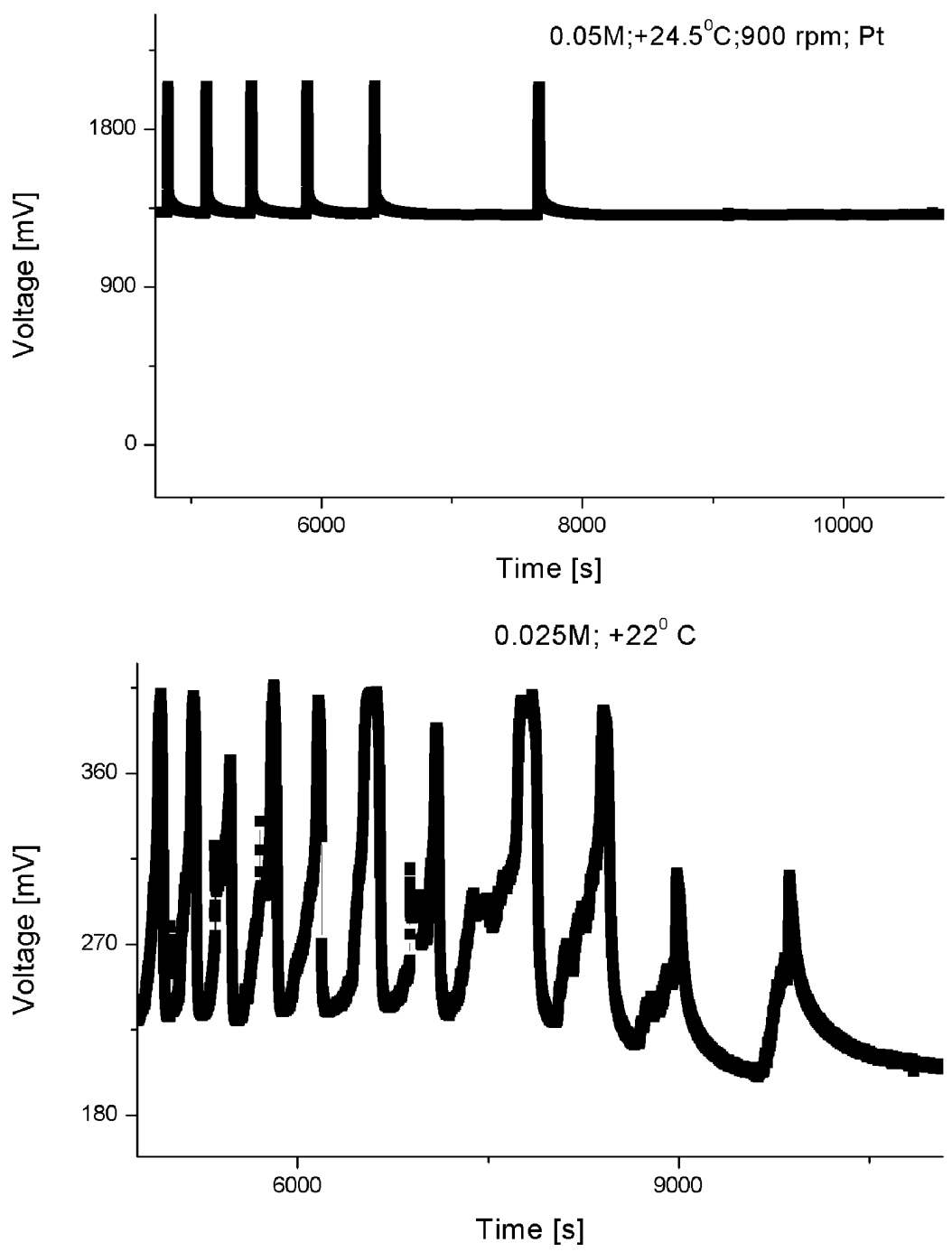

Fig. 13. Result of the experiment from Fig. 12, recorded by a Pt electrode.
Fig. 14. The same experiment as in Figs. 17, 18 but at higher temperature. runs the release of $\mathrm{CO}_{2}$ during the reactions has not been measured in time. Hence we can not say how the way of releasing the $\mathrm{CO}_{2}$ is. One could believe that it is the oscillation way. But it can not be true as was stated in [13] although for not exactly the same substrates as in our case.

\subsection{Effect of Oxygen}

In some experimental conditions without a nitrogen flow over the chemical mixture it is possible that the destructive effect of oxygen disturbs the sequential oscillations. On the other hand, nitrogen flow affects sequential oscillations, as is discussed in the next chapter. The effect of oxygen from air seems to be greater (in removing sequential oscillations) than the effect of flowing nitrogen, i.e., the effect as expressed by the number of sequential oscillations appearing in the two kinds of experiments. A greater number of sequential oscillations was observed in the case of flowing nitrogen than in the case of flowing air, namely 11 sequential oscillations were observed with flowing nitrogen in the first part of Table 1, no sequential oscillations were observed for flowing air and 6 sequential oscillations in no flowing air (i. e. in air, simply speaking), as we stated above. When attaining an equilibrium, oscillatory peaks have got a more relaxed character in some experiments with flowing nitrogen and air than in oxygen. Flowing air destroys oscillations after ca. $2 \mathrm{~h}$ (experimental conditions from the first line of Table 1). The same situation was seen for flowing oxy- 

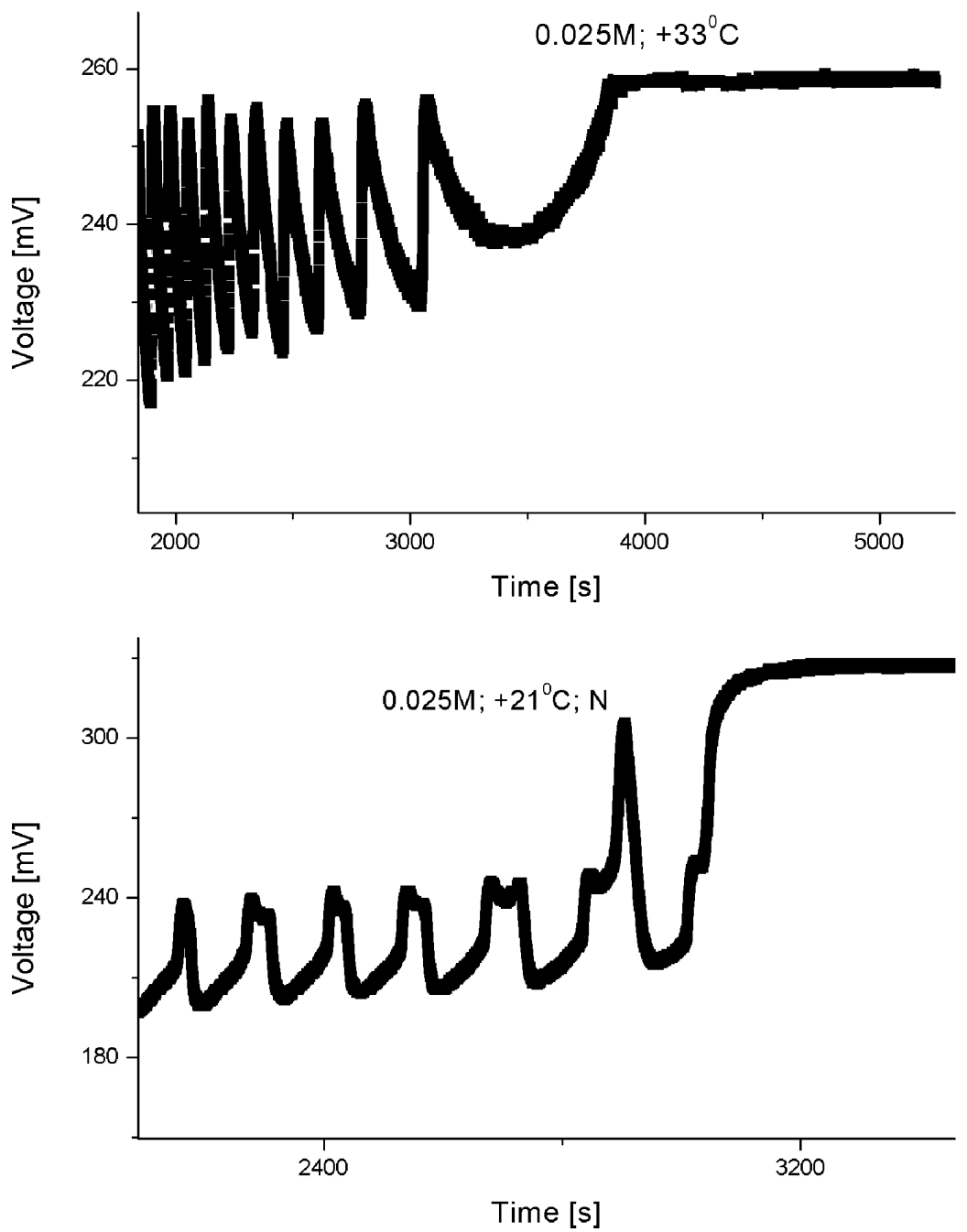

Fig. 15. The experiment from Figs. $14,17,18$ but at the highest temperature. gen, whereas in an experiment with flowing nitrogen some oscillations ceased completely after ca. $3 \mathrm{~h}$.

\section{Discussion}

We could observe that at $10 \%$ of oxygen or more and for an initial concentration of malonic acid [MA $]_{0}$ between $0.15 \mathrm{M}$ and $0.6 \mathrm{M}$ the system attains an equilibrium by some of mixed mode oscillations. Such a behaviour of the system could be described by a model used recently.

Some sequential oscillations appeared close to the equilibrium condition of the chemical reaction. They were observed in the experiment for $[\mathrm{MA}]_{0}=0.1 \mathrm{M}$ or less at $0-20 \%$ of oxygen over the chemical mixture. $\mathrm{CO}_{2}$ (one of some gaseous products of the reaction and the main one) can destroy some concentrations' oscillations for a given concentration of reagents.

The concentration of $\mathrm{CO}_{2}$ in the solution increases during the reaction. If there is a great amount of $\mathrm{CO}_{2}$ in the solution, greater than the solubility of $\mathrm{CO}_{2}$ (for the given experimental conditions), $\mathrm{CO}_{2}$ must be released which happens. Such a large amount of $\mathrm{CO}_{2}$ disturbs the oscillations and the $\mathrm{CO}_{2}$ 's release causes the appearance of some oscillations. Such events are referred to as sequential oscillations. The chemical potential of $\mathrm{CO}_{2}$, as a product of the reaction, is directed in the opposite direction to chemical potentials of some substrates. Therefore, at very low concentrations of malonic acid, those chemical potentials may give a virtual chemical equilibrium (seen as a break between oscillations). Obviously, the chemical mixture, saturated with 

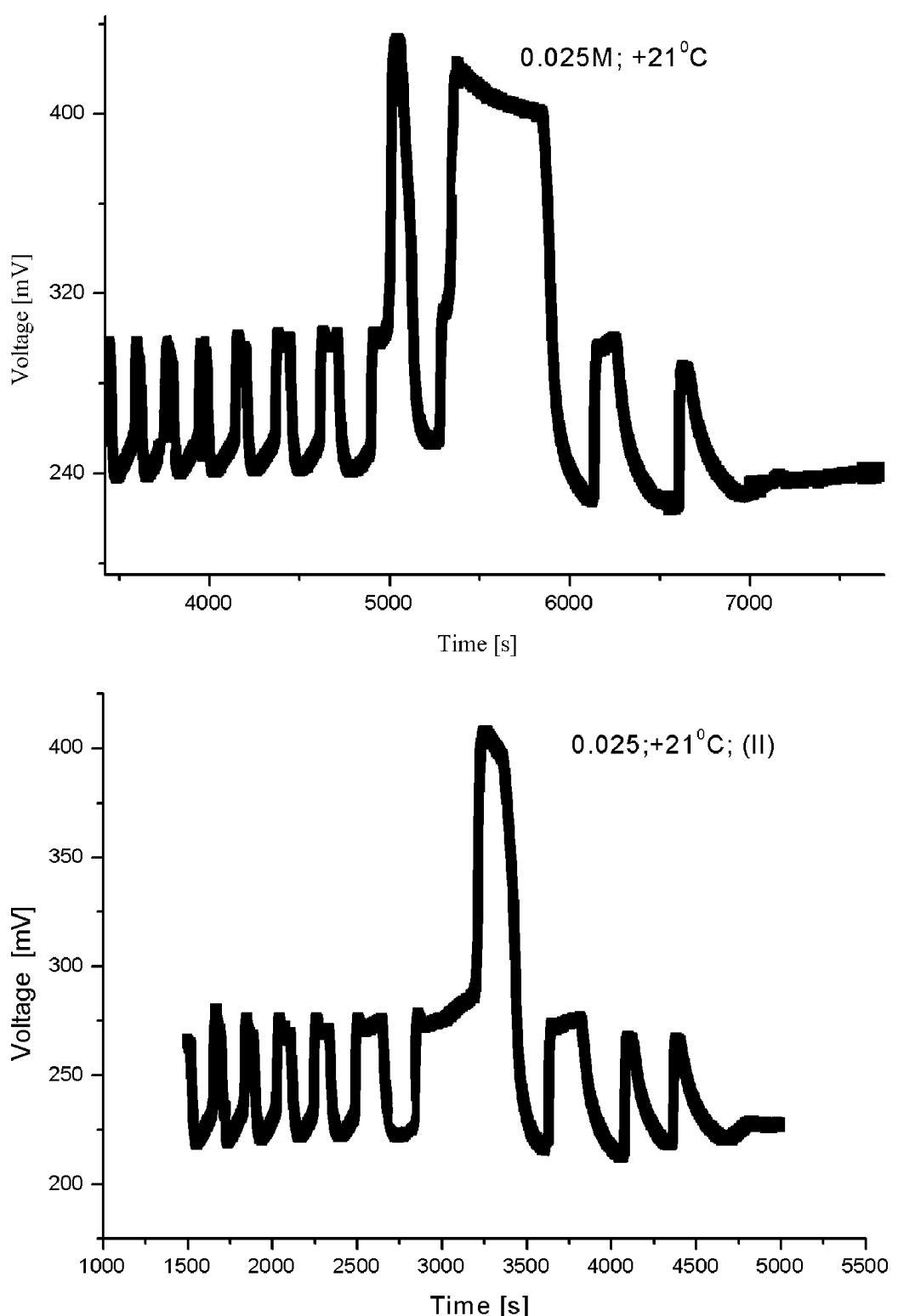

Fig. 17. Result for an experiment performed as in Fig. 16 but with air.
Fig. 18. The same experiment as in Fig. 13 (for showing a reproducibility).
$\mathrm{CO}_{2}$, is very unstable. It is sufficient for the temperature to change within the limit of experimental error or for the stirring rate to change within the limit of experimental error for the reactive solution to eject the non-soluble amount of $\mathrm{CO}_{2}$ thereby causing some oscillations (seen in the experiment) as sequential oscillations.

Additionally, increasing the temperature decreases the amount of $\mathrm{CO}_{2}$ in solution, hence sequential oscillations disappear (Fig. 6). The behaviour of the system with increasing temperature (disappearing of sequen- tial oscillations) may be confirmed by the experiment described in item 2) above. In this experiment (electrodes in a cover) $\mathrm{CO}_{2}$ had only a little volume for escape. The appearance of some sequential oscillations occurred for $0.025 \mathrm{M}$ and $0.03 \mathrm{M}[\mathrm{MA}]_{0}(0.00388 \mathrm{M}$ [ferroin $]_{0}$, the other concentrations as above), whereas in the same experiment, but with electrodes inserted as normal in the batch system (large volume for $\mathrm{CO}_{2}$ escape), no sequential oscillations were observed (of course for the same chemical system). The electrode cover inhibited $\mathrm{CO}_{2}$ escape destroying some oscilla- 
tions over a rather long period of time. When the pressure of $\mathrm{CO}_{2}$ increased beyond its solubility, $\mathrm{CO}_{2}$ was released. Sequential oscillations appeared appropriate for the reagent concentrations (substrates, existing still in the solution).

Let's note that some sequential oscillations follow a great value of electrode's potential in the experiment of 2), whereas they follow its low value in most experiments (electrodes inserted not in the cover).

Increasing the stirring rate practically removes sequential oscillations because stirring removes a portion of the gaseous products existing in the solution.

Some experiments performed under the same conditions as above, but in a new reactor with a diameter about 1.5 times smaller, showed that the time needed to attain an equilibrium was shortened several times. It can be connected with smaller efficiency of mixing, i. e. the mixing rate in the small reactor is in reality smaller than in the big one although the same stirring rates $(740 \mathrm{rpm})$ were used in both cases. And the smaller stirring rate causes of more gaseous products to be left in the solution which disturbs the reaction and results in shortening of the time of reaching an equilibrium. We have not described that experiment in the experimental part of the work, because it has not been strongly connected with some experiments showing an evidence of some mixed mode, sequential oscillations and relaxation oscillations which are the main subject of considerations in the article.

The article [14] tries to explain the stirring effect by dependence of a kinetic constant on stirring rate, dimensions of the stirring bar, etc. The efficiency of stirring has been not explicitly included into those considerations [14]. We are convinced that the efficiency of stirring is responsible for shortening by several times the total time of our reaction performed in a reactor with a smaller diameter (all other experimental conditions remaining the same). This efficiency is connected with better or worse removing of gaseous products of the reaction as we stated above.

Explanation of sequential oscillations [7] by two BZ reaction processes, i. e., between radicals and by means of $\mathrm{Br}^{-}$control, seems not to be useful in our case. This is because some sequential oscillations from [7] could not be observed without removing gaseous oxygen, present (in small amounts) in solutions of the substrates (prior to the reaction). In our case all experimental results shown above were obtained without removing oxygen from substrates' solutions before the experiment.
We try to describe the problem of oscillations death seen by a platinum electrode and later oscillations death registered by $\mathrm{Br}$ electrode in the following way.

One can assume that a too large amount of $\mathrm{CO}_{2}$ destroys the two signals (those of the $\mathrm{Pt}$ and $\mathrm{Br}$ electrodes) and a smaller amount of $\mathrm{CO}_{2}$ destroys only the Pt signals. Such an unusual behaviour of the BZ reaction was observed many times in a batch reactor following CSTR measurements. In such a situation the CSTR reactor was changed into a batch reactor. We observed that a rising $\mathrm{CO}_{2}$ level (observed from the position of a drop of solution in a small tube inserted in the cover) produced further oscillations. The problem has been still waitingfor any more detailed explanation.

\section{Conclusions}

Volatile products of the reaction can either break the reaction (lack of oscillations), cause relaxation peaks or cause time-delayed Hopf bifurcations [1]. The sequential oscillations observed in the experiment appeared close to the equilibrium state of the subject chemical reaction. $\mathrm{CO}_{2}$, one of the reaction products, can quench oscillations at specific (for the event) concentrations of the reagents. If there is a large amount of $\mathrm{CO}_{2}$ and other volatile products in the solution, greater than their solubility concentration, they are released. Excessive amounts of volatile products disturb the oscillations resulting in the disappearance of some oscillations. Changes of temperature or stirring rate, even within the limits of experimental error as well as a change of electrode position during the measurement can be a driving force for a quick release of such products. In the case of flowing nitrogen a smaller amount of $\mathrm{CO}_{2}$ remains in the solution. This result is in accordance with Henry's law. Therefore, the number of sequential oscillations increases in such an experiment in comparison with the same experiment (the same initial concentrations and other conditions) carried out in air. Biological similarities to such an unusual behaviour are easy to find. Namely in some cases of clinical death (no breathing for some time), life (restored breathing) can be 'switched on' without any outside help.

\section{Acknowledgement}

The possibility to present the article during the Conference "Dynamic Days 2005" (25-28 July 2005, Berlin) is fully acknowledged by the author. 
[1] Y. Y. Kalishyn, M. Rachwalska, V. O. Khavrus, and P. Strizhak, Phys. Chem. Chem. Phys. 7, 1680 (2005).

[2] A. L. Kawczyński and P.E. Strizhak, J. Chem. Phys. 112, 6122 (2000).

[3] A. L. Kawczyński, V. O. Khavrus, and P.E. Strizhak, Chaos 10, 299 (2000).

[4] M. Rachwalska and A. L. Kawczyński, J. Phys. Chem. A 103, 3455 (1999).

[5] L. D. Hall and J. C. Waterton, J. Am. Chem. Soc. 13, 101 (1979).

[6] M. Wittmann, P. Stirling, and J. Bódiss, Chem. Phys. Lett. 141, 241 (1987).

[7] G.P. Misra, R.P. Washington, and J.A. Pojman, J. Phys. Chem. A 102, 612 (1998).
[8] H. Li and X. Huang, Chem. Phys. Lett. 255, 137 (1996).

[9] L. Adamciková, Z. Farbulová, and P. Ševcik, New J. Chem. 25, 487 (2001).

[10] L. Adamciková, Z. Farbulová, P. Ševcik, and A. L. Kawczyński, J. Phys. Chem. A 107, 508 (2003).

[11] J. C. Wang, F. Hynne, P. G. Sorensen, and K. Nielsen, J. Phys. Chem. 100, 17593 (1996).

[12] B. Z. Shakhashiri and G. Gordon, Inorg. Chem. 7, 2454 (1968).

[13] P. Sevcik, D. Misicak, and L. Adamcikova, CHEM PAP - Chem. Zvesti 60, 1 (2006).

[14] Z. Noszticzius, Z. Bodnár, L. Garamszegi, and M. Wittmann, J. Phys. Chem. 95, 6575 (1991). 